\title{
Chemical Evolution of Strongly Magnetized Quark Core in a Newborn Neutron Star
}

\author{
Tanusri Ghosh ${ }^{a) *}$ and Somenath Chakrabarty ${ }^{a, b) \dagger}$ \\ a) Department of Physics, University of Kalyani, West Bengal 741 235, India \\ b) Inter-University Centre for Astronomy and Astrophysics, Post Bag 4 \\ Ganeshkhind, Pune 411 007, India
}

(August 29, 2021)

PACS:12.38.Mh, 12.15.Ji, 95.30.Cq, 97.60.Jd

\begin{abstract}
The chemical evolution of nascent quark matter core in a newborn compact neutron star is studied in presence of a strong magnetic field. The effective rate of strange quark production in degenerate quark matter core in presence of strong magnetic fields is obtained. The investigations show that in presence of strong magnetic fields a quark matter core becomes energetically unstable and hence a deconfinement transition to quark matter at the centre of a compact neutron star
\end{abstract}

\footnotetext{
*E-mail:tanusri@klyuniv.ernet.in

${ }^{\dagger}$ E-mail:somenath@klyuniv.ernet.in
} 
under such circumstances is not possible. The critical strength of magnetic field at the central core to make the system energetically unstable with respect to dense nuclear matter is found to be $\sim 4.4 \times 10^{13} \mathrm{G}$. This is the typical strength at which the Landau levels for electrons are populated. The other possible phase transitions at such high density and ultra strong magnetic field environment are discussed. 


\section{INTRODUCTION}

More than a decade ago, it was suggested by Witten [1] that a flavor symmetric mixture of $u, d$ and $s$-quarks known as strange quark matter (SQM) would be the most stable configuration of hadronic matter. An extensive theoretical investigations on the stability of both bulk as well as quasi-bulk properties of SQM using MIT bag model and also with some other confinement models have proved the reality of such speculation [2 [4]. Usually the strange quark is attributed a minor role in the standard model of the Universe. However, it has also been suggested by Witten that strange quark matter might be an important relic from the early Universe and also a constituent of neutron stars. Although strange quark matter could be absolutely stable and thus be the true ground state of hadronic matter, long-lived or stable strange quarks are only found in the regions with very high density, otherwise, the relatively large $s$-quark mass and the weak mixing to the down quarks lead to rapid decay. In a quark matter system, either at the core of a compact neutron star or in the early Universe-microsecond after big bang, s-quarks are produced through a number of weak processes. It is generally believed that microsecond after big bang, the Universe was completely filled with a hot and dense soup of quark matter. The survival of strange quark nuggets relic till today from the early Universe is a controversial issue [6]. It is also expected that a deconfinement transition to bulk quark matter may cur at the core of a neutron star if the density exceeds a few times normal nuclear matter density. In such a cold quark matter system, s-quarks are produced through weak processes. Although the existence of strange quark nuggets relic produced as a result of first order QCD phase transition in the early Universe is highly uncertain, the presence of exotic quark matter core in a compact neutron 
star can not be ruled out. A few years ago, Madsen and Hiselberg et. al. have investigated with detail numerical calculations the rate of $s$-quark production in dense quark matter in the context of survival of strange quark nuggets produced at the time of primordial QCD phase transition [6,7]. Recently, Glendenning and his group have reported a lot of exciting results on the existence of strange quark matter core in a compact neutron star and its effect on various gross-properties of hybrid stars [8]. It has also been suggested that a transition to quark matter core may occur during slowing down of a old neutron star 9]. A first order phase transition to quark matter at the core of a neutron star is also possible by seeding strange droplets from outside and by neutrino sparking [10. However, in none of these papers, the effect of strong magnetic field of the neutron star (particularly in the case of newborn neutron star) on such exotic matter have been considered. Large magnetic fields are known to be present in neutron stars [11 [13]. The direct evidence from pulsar observations show that the strength of this magnetic field at the neutron star surface is $10^{12}-10^{14} \mathrm{G}$. Then it can very easily be shown by the scalar virial theorem that magnetic field strength at the core region of a neutron star may reach up to $10^{18}-10^{19} \mathrm{G}$ [14. On the other hand, from the stability criteria of a compact magnetized neutron star it can very easily be shown following the famous work by Chandrasekhar and Fermi [15] in the context of magnetized white dwarfs, that the upper limit for the strength of magnetic field at the core region is $\sim 10^{19} \mathrm{G}$. The presence of such strong magnetic field inside newborn neutron stars significantly modifies the order of qaurk-hadron phase transition at the core. It has been shown that a first order quark-hadron phase transition initiated by the nucleation (by thermal or quantum fluctuation) of quark droplets in a system of 
meta-stable neutron matter in presence of a strong magnetic field $\left(\geq B_{m}^{(c)(e)}\right)$ is absolutely forbidden [5,16]. However, some higher order transitions, e.g. metalinsulator type of second order phase transition is allowed if the strength of magnetic field is $\leq 10^{20} \mathrm{G}$. Which is of course too high to achieve at the core of a neutron star. Even if the transition is of second order in nature, the gross properties of dense quark matter are considerably modified with respect to the field free case. The presence of strong magnetic field also affects the weak and electro-magnetic processes which may occur in the degenerate quark matter core [17]. The presence of strong magnetic field shifts the $\beta$-equilibrium point of the quark matter system [18]. It also affects the abundances of various components in $\beta$-equilibrium. The photon emission by bremsstrahlung, photon splitting and neutrino emission processes are significantly affected by the presence of strong magnetic field 17, 19. It is also believed that pulsar kicks are generated by the asymmetric neutrino emission in presence of strong magnetic fields 20 23]. In a dense quark matter, since $s$-quarks are produced or annihilated through weak processes, the presence of strong magnetic field should also alter the production and decay rates of $s$-quarks. To the best of our knowledge, theoretical investigation of this particular problem has not been reported earlier. In the previous work by Madsen and Hiselberg et. al. [6,7] the rate of $s$-quark production in degenerate cold quark matter $(T<10 \mathrm{MeV})$ was studied for the field free case in the context of stability of strange nuggets as the relic of big bang deconfinement transition. In the present paper we shall study the effect of strong magnetic field on the net rates of $u, d$ and $s$-quarks production and there by study the chemical evolution of nascent degenerate quark matter core in a compact neutron star. We shall also consider the effect of neutrino trapping in degenerate 
quark matter on the chemical evolution in presence of a strong magnetic field. The main finding of this paper is that the chemical evolution of nascent quark matter core in presence of a strong magnetic field leads to an unphysical scenario- the final state in $\beta$-equilibrium is energetically unstable with respect to the normal hadronic matter of neutron star. Although a flavor symmetric mixture of $u, d$ and $s$-quarks at zero temperature is energetically much more stable than the corresponding field free case, the chemical evolution of a bulk system consisting of $u, d$ and $s$-quarks (not a flavor symmetric mixture) shows that the quark matter core of a newborn neutron star may become energetically unstable if the magnetic field strength exceeds a typical value $\sim 4.4 \times 10^{13} \mathrm{G}$, which is the strength of magnetic field at which the Landau levels for electrons are populated and quantum mechanical effect of strong magnetic field on electrons become important. Since the formation of a flavor symmetric quark matter at the central region of a compact neutron star is possible only through the chemical evolution of ordinary quark matter or $u, d$ and $s$ flavor asymmetric quark matter, it would really be interesting to investigate the time evolution of various species in the system both for field free case and in presence of a strong magnetic field.

For the sake of simplicity we neglect quark-quark interaction in the degenerate quark matter. The temperature of the system is taken to be $1 \mathrm{MeV}\left(\simeq 10^{9} \mathrm{~K}\right)$. The increase or decrease in temperature of the system causes an enhancement or suppression of effective production rate of $s$-quark respectively. However, it only makes a quantitative change in our result. The qualitative nature remains unaltered. It is further assumed that the external magnetic field $B_{m}$ is constant throughout the matter. The convenient choice of gauge is $A_{0}=0$ and $\vec{A}=\left(0, x B_{m}, 0\right)$ corresponding 
to the constant magnetic field $B_{m}$ along z-axis. If the strength of magnetic field $B_{m}$ is greater than the critical value $B_{m}^{(c)(i)}=m_{i}^{2} / q_{i}$ for the $i$-th charged particle, where $m_{i}$ and $q_{i}$ are respectively the mass and charge of the $i$-th species, the corresponding single particle energy is given by $\varepsilon_{i}=\left(p_{z}^{2}+m_{i}^{2}+2 q_{i} \nu B_{m}\right)^{1 / 2}$, otherwise the usual expression for field free $\left(B_{m}=0\right)$ case is used, where $\nu$ is the Landau quantum number. The critical strength of magnetic field as mentioned above can be obtained by equating the cyclotron quantum with the rest mass energy of the $i$-th charged particle. If the magnetic field strength exceeds this typical value, the quantum mechanical effect of strong magnetic field on the $i$-th charged component becomes important. This is known as Landau diamagnetism. For electrons of mass $0.5 \mathrm{MeV}$, this typical strength is $B_{m}^{(c)}(e) \simeq 4.4 \times 10^{13} \mathrm{G}$, whereas for $u$ and $d$-quarks of current mass $5 \mathrm{MeV}$, it is $\simeq 10^{2} B_{m}^{(c)(e)}$. On the other hand for $s$-quark of current mass $150 \mathrm{MeV}$, this typical value is $\sim 10^{20} \mathrm{G}$. Which is too high to achieve even at the core of a newborn neutron star. Therefore in the case of $s$-quarks, quantum mechanical effect of strong magnetic field is insignificant and results for the field free case are used.

The paper is organized in the following manner, in section 2 we have given the basic formalism to study the chemical evolution of compact and degenerate nascent quark matter core with non-degenerate neutrinos (i.e. neutrinos are assumed to leave the system freely immediately after their formation), both in absence and presence of strong magnetic fields. In the case of $B_{m} \neq 0$, we have considered two possible scenarios-(i) magnetic field strength is low enough so that only electrons are affected and (ii) the field strength is relatively stronger so that all the charged particle components except $s$-quarks are affected. In section 3 we have discussed with 
detail numerical calculations, the effect of neutrino trapping on chemical evolution of the system for both $B_{m}=0$ and $B_{m} \neq 0$ cases. Concluding remarks are presented in section 4 .

\section{BASIC FORMALISM: WITH NON-DEGENERATE NEUTRINOS}

To study the chemical evolution of degenerate quark matter at the core of a newborn neutron star we consider the following weak interaction processes which are taking place at the quark matter core

$$
\begin{aligned}
& d \rightarrow u+e^{-}+\bar{\nu}_{e} \\
& u+e^{-} \rightarrow d+\nu_{e} \\
& s \rightarrow u+e^{-}+\bar{\nu}_{e} \\
& u+e^{-} \rightarrow s+\nu_{e} \\
& u+d \rightleftharpoons u+s
\end{aligned}
$$

We further assume that the system is charge neutral and produced neutrinos/antineutrinos leave the system freely (i.e. they are non-degenerate). The approach to chemical equilibrium of the system is governed by the following sets of kinetic equations

$$
\frac{d Y_{u}}{d t}=\frac{1}{n_{B}}\left[\Gamma_{1}-\Gamma_{2}+\Gamma_{3}-\Gamma_{4}\right]
$$




$$
\frac{d Y_{d}}{d t}=\frac{1}{n_{B}}\left[-\Gamma_{1}+\Gamma_{2}-\Gamma_{5}+\Gamma_{6}\right]
$$

Baryon number conservation and charge neutrality conditions relate strange quark and electron abundances with that of $u$ and $d$-quark and are given by

$$
\begin{gathered}
Y_{s}=3-Y_{u}-Y_{d} \\
Y_{e}=Y_{u}-1
\end{gathered}
$$

where $Y_{i}=n_{i} / n_{B}$ is the abundance for the $i$-th component $\left(i=u, d, s, e\right.$ and $\nu_{e}$ (for the degenerate neutrino case only)), $n_{B}$ is the baryon number density, $\Gamma_{i}$ is the rate of $i$-th weak interaction process $(i=1, \ldots, 5)$ and $\Gamma_{6}$ is the rate of reverse process as indicated by eqn.(5).

To study chemical evolution of the system we solve the differential eqns.(6) and (7) numerically along with two constraint equations, eqns.(8) and (9) and use the rates $\Gamma_{1}$ to $\Gamma_{6}$, appear on the right hand side of eqns.(6) and (7). Since these differential equations are of first order in nature, we need at least one initial condition for both $Y_{u}$ and $Y_{d}$ in order to get the complete solutions. For a neutron star of mass $\simeq 1.4 M_{\odot}$ the baryon number density $n_{B}$ at the centre is of the order of $3-4 n_{0}$, temperature $\sim 10^{9} \mathrm{~K}$ and proton content is about $4 \%$. Then the initial conditions are given by $Y_{u}(t=0)=1.04, Y_{d}(t=0)=1.96$. As a consequence of baryon number and charge conservation we have $Y_{s}(t=0)=0$ and $Y_{e}=0.04$.

\section{Weak Reaction Rates: $B_{m}=0$}

In this subsection we shall first derive the rates $\left(\Gamma_{1}\right.$ to $\left.\Gamma_{5}\right)$ for the processes given by eqns.(1)-(5) and also the rate $\Gamma_{6}$ for the reverse process as indicated by eqn.(5), for the field free case. 
The reaction rate for the process (1) is given by

$$
\begin{aligned}
\Gamma_{1}= & 6 \times 64 G^{2} \cos ^{2} \theta_{c} \int \sum_{i=1}^{4} \frac{d^{3} p_{i}}{(2 \pi)^{3} 2 \varepsilon_{i}}(2 \pi)^{4} \delta^{4}\left(p_{1}-p_{2}-p_{3}-p_{4}\right) \\
& f\left(\varepsilon_{1}\right)\left(1-f\left(\varepsilon_{3}\right)\right)\left(1-f\left(\varepsilon_{4}\right)\right)\left(p_{1} \cdot p_{2}\right)\left(p_{3} . p_{4}\right)
\end{aligned}
$$

where $i=1,2,3,4$ stand for $d, \bar{\nu}_{e}, u$ and $e$ respectively, $f\left(\varepsilon_{i}\right)=1 /(1+$ $\exp \left(\left(\varepsilon_{i}-\mu_{i}\right) / T\right)$, the equilibrium (Fermi) distribution function for the $i$-th component and $p_{i}, \varepsilon_{i}, \mu_{i}$ are respectively the 4-momentum, energy and chemical potential of the particle $i, \mathrm{G}$ is the weak coupling constant, $\theta_{c}$ is the Cabibbo angle $\left(\cos ^{2} \theta_{c} \simeq 0.74\right)$ and $\mathrm{T}$ is the temperature of the system. The angular part of the integrals can be evaluated without any approximation [6, [4] and we get

$$
\begin{aligned}
\Gamma_{1}= & \frac{12 G^{2}}{\pi^{6}} \cos ^{2} \theta_{c} \int \sum_{i=1}^{4} d \varepsilon_{i} \delta\left(\varepsilon_{1}-\varepsilon_{2}-\varepsilon_{3}-\varepsilon_{4}\right) \\
& f\left(\varepsilon_{1}\right)\left(1-f\left(\varepsilon_{3}\right)\right)\left(1-f\left(\varepsilon_{4}\right)\right) I_{n}\left(\varepsilon_{1}, \varepsilon_{2}, \varepsilon_{3}, \varepsilon_{4}\right)
\end{aligned}
$$

where

$$
\begin{aligned}
& I_{n}\left(\varepsilon_{1}, \varepsilon_{2}, \varepsilon_{3}, \varepsilon_{4}\right)=k_{-}^{12} k_{+}^{34} I_{1}\left(\varepsilon_{1}, \varepsilon_{2}, \varepsilon_{3}, \varepsilon_{4}\right)+\frac{1}{6}\left(k_{+}^{34}-k_{-}^{12}\right) I_{2}\left(\varepsilon_{1}, \varepsilon_{2}, \varepsilon_{3}, \varepsilon_{4}\right)- \\
& \frac{1}{20} I_{3}\left(\varepsilon_{1}, \varepsilon_{2}, \varepsilon_{3}, \varepsilon_{4}\right) \\
& k_{ \pm}^{i j}=\varepsilon_{i} \varepsilon_{j} \pm \frac{1}{2}\left(p_{i}^{2}+p_{j}^{2}\right) \\
& \begin{aligned}
I_{n}\left(\varepsilon_{i}, \varepsilon_{j}, \varepsilon_{k}, \varepsilon_{l}\right)= & \left(P_{i j}^{2 n-1}-p_{i j}^{2 n-1}\right) \theta\left(P_{k l}-P_{i j}\right) \theta\left(p_{i j}-p_{k l}\right) \\
& +\left(P_{i j}^{2 n-1}-p_{k l}^{2 n-1}\right) \theta\left(P_{k l}-P_{i j}\right) \theta\left(p_{k l}-p_{i j}\right) \theta\left(P_{i j}-p_{k l}\right) \\
& +\left(P_{k l}^{2 n-1}-p_{k l}^{2 n-1}\right) \theta\left(P_{i j}-P_{k l}\right) \theta\left(p_{k l}-p_{i j}\right) \\
& +\left(P_{k l}^{2 n-1}-p_{i j}^{2 n-1}\right) \theta\left(P_{i j}-P_{k l}\right) \theta\left(p_{i j}-p_{k l}\right) \theta\left(P_{k l}-p_{i j}\right)
\end{aligned}
\end{aligned}
$$


$P_{i j}=p_{i}+p_{j}, p_{i j}=\left|p_{i}-p_{j}\right|, p_{i}=\left|\vec{p}_{i}\right|$ is the magnitude of three momentum vector and $\varepsilon_{i}=\left(p_{i}^{2}+m_{i}^{2}\right)^{1 / 2}$ (we assume $\left.\hbar=c=k_{B}=1\right)$.

In the case of a compact neutron star, the central density is very high $\left(\sim 3-4 n_{0}\right.$ to have a deconfinement transition) but the temperature is low enough $\left(\sim 10^{9} \mathrm{~K}\right)$, as a consequence $u, d$ quarks and electrons are relativistic in nature and are strongly degenerate. Under such an extreme condition all the integrals except one in eqn.(11) can be evaluated analytically and the final expression for rate of the process (1) is given by

$$
\Gamma_{1}\left(\mu_{d}, \mu_{u}, \mu_{e}, T\right)=\frac{3 G^{2}}{4 \pi^{5}} \cos ^{2} \theta_{c} \int_{0}^{\infty} d x \frac{\left(x-\xi_{d}\right)^{2}+\pi^{2}}{1+\exp \left(x-\xi_{d}\right)} I\left(\mu_{d}, T x, \mu_{u}, \mu_{e}\right)
$$

where in the expression for $I$ we have replaced energy and momentum of all the particles except the neutrino by their values on the Fermi surface, i.e. we put $\varepsilon_{i}=\mu_{i}$ and $p_{i}=p_{F_{i}}=\left(\mu_{i}^{2}-m_{i}^{2}\right)^{1 / 2}$, we have also changed the integration variable $\varepsilon_{\nu}$ to $x$, where neutrino energy $\varepsilon_{\nu}=p_{\nu}=x T$ and $\xi_{d}=\left(\mu_{d}-\mu_{u}-\mu_{e}\right) / T$ which is zero in the $\beta$-equilibrium condition. Following the same methodology, we have obtained the rate for the process (2), given by

$$
\Gamma_{2}\left(\mu_{d}, \mu_{u}, \mu_{e}, T\right)=\frac{3 G^{2}}{4 \pi^{5}} \cos ^{2} \theta_{c} T^{3} \int_{0}^{\infty} d x \frac{\left(x+\xi_{d}\right)^{2}+\pi^{2}}{1+\exp \left(x+\xi_{d}\right)} J\left(\mu_{d}, T x, \mu_{u}, \mu_{e}\right)
$$

where

$$
\begin{aligned}
J\left(\varepsilon_{1}, \varepsilon_{2}, \varepsilon_{3}, \varepsilon_{4}\right)= & k_{+}^{12} K_{+}^{34} I_{1}\left(\varepsilon_{1}, \varepsilon_{2}, \varepsilon_{3}, \varepsilon_{4}\right) \\
& -\frac{1}{6}\left(k_{+}^{34}+k_{+}^{12}\right) I_{2}\left(\varepsilon_{1}, \varepsilon_{2}, \varepsilon_{3}, \varepsilon_{4}\right)+\frac{1}{20} I_{3}\left(\varepsilon_{1}, \varepsilon_{2}, \varepsilon_{3}, \varepsilon_{4}\right)
\end{aligned}
$$

The reaction rates for the processes (3) and (4) corresponding to $s$-quark can very easily be evaluated by replacing all the parameters for $d$-quarks by the corresponding 
$s$-quark values (i.e. $\mu_{d} \rightarrow \mu_{s}, \xi_{d} \rightarrow \xi_{s}, m_{d} \rightarrow m_{s}$ ) and $\cos ^{2} \theta_{c}$ by $\sin ^{2} \theta_{c}$ in the expressions for $\Gamma_{1}$ and $\Gamma_{2}$ respectively.

For the weak process (5) we have

$$
\begin{aligned}
\Gamma_{5}\left(\mu_{d}, \mu_{u}, \mu_{s}, T\right)= & \frac{9 G^{2}}{2 \pi^{5}} \cos ^{2} \theta_{c} \sin ^{2} \theta_{c} T^{3} \int_{-\infty}^{\left(\mu_{s}-m_{s}\right) / T} d x \\
& \frac{\left(x+\xi_{s}\right)^{2}+\pi^{2}}{(1+\exp (x))\left(1+\exp \left(-x_{x}-\xi_{s}\right)\right)} J\left(\mu_{d}, \mu_{u}, \mu_{u}, \mu_{s}-T x\right)
\end{aligned}
$$

where $\xi_{s}=\left(\mu_{d}-\mu_{s}\right) / T$.

The reaction rate for the reverse process as shown in eqn.(5) is related to the forward rate $\Gamma_{5}$ by

$$
\Gamma_{6}=\exp \left(-\xi_{s}\right) \Gamma_{5}
$$

\section{Weak Reaction Rates: $B_{m} \neq 0$}

We shall now evaluate the rates $\Gamma_{1}$ to $\Gamma_{6}$ in presence of strong magnetic fields. We consider two possible cases: the magnetic field $B_{m}$ is not very high $\left(B_{m}^{(c)(e)}<\right.$ $\left.B_{m}<B_{m}^{(c)(u, d)}\right)$ so that the quantum mechanical effect is important for electrons only, and finally the magnetic field is strong enough to affect all the charged particle components except $s$ - quarks $\left(B_{m}^{(c)(u, d)}<B_{m}<B_{m}^{(c)(s)}\right)$.

Let us first consider case 1. The magnetic field $B_{m}^{(c)(e)}<B_{m}<B_{m}^{(c)(u, d)}$. To evaluate the rates for the weak processes (1)-(4), we use the modified form of spinor solution of Dirac equation in presence of a strong magnetic field for electron [16,24,25]. For other charged particles, the usual plane wave Dirac spinor solutions for field free case are used. Since only electrons are affected, the rates for the process (5) and its reverse process will remain unchanged. 
In this case the rate of the process (1) is given by

$$
\begin{aligned}
\Gamma_{1}= & \frac{3 G^{2} q_{e} B_{m}}{2 \pi^{6}} T^{4} \cos ^{2} \theta_{c} \sum_{\nu=0}^{\infty} \mu_{u} \mu_{e}\left(\frac{p_{F_{u}}}{p_{F_{e}}}\right) \\
& \int_{-\infty}^{+\infty} \int_{-\infty}^{+\infty}\left(x_{u}+x_{e}-\frac{\mu_{u}+\mu_{e}-\mu_{d}}{T}\right)^{2} f\left(x_{u}\right) f\left(x_{e}\right) d x_{u} d x_{e}
\end{aligned}
$$

where $q_{e}$ is the charge carried by electron, $f\left(x_{i}\right)=1 /\left(1+\exp \left(x_{i}\right)\right)$, and $p_{F_{e}}=$ $\left(\mu_{e}^{2}-m_{e}^{2}-2 \nu q_{e} B_{m}\right)^{1 / 2}$ is the Fermi momentum for electron. The sum $\nu$ is over Landau quantum number for the electron which in principle can have all possible positive integer values including zero.

Similarly the rate for the process (2) is given by

$$
\begin{aligned}
\Gamma_{2}= & \frac{3 G^{2}}{2 \pi^{6}}\left(q_{e} B_{m}\right) T^{4} \cos ^{2} \theta_{c} \sum_{\nu=0}^{\infty} \mu_{u} \mu_{e}\left(\frac{p_{F_{u}}}{p_{F_{e}}}\right) \int_{-\infty}^{+\infty} \int_{-\infty}^{+\infty} d x_{u} d x_{e} \\
& \left(x_{u}+x_{e}+\frac{\mu_{u}+\mu_{e}-\mu_{d}}{T}\right)^{2} f\left(x_{u}\right) f\left(x_{e}\right)
\end{aligned}
$$

As before the rates for the processes (3) and (4) may be obtained by simply replacing all the parameters for $d$-quarks by the corresponding $s$-quark values and $\cos ^{2} \theta_{c}$ by $\sin ^{2} \theta_{c}$ in eqns.(20) and (21) respectively. Whereas the rates for the direct and the reverse processes given by eqn.(5) remain unaltered.

Next we consider the second case possibility, $B_{m}^{(c)(s)}>B_{m}>B_{m}^{(c)(u, d)}$. In this case modified spinor solutions for all the charged particle components except $s$-quark are used to evaluate rates of the weak processes [16,24,25]. As we have noticed that compact analytical expressions for the rates can only be obtained if we assume that zeroth Landau levels are populated for the magnetically affected charged species. Otherwise we have to evaluate the rates numerically right from the beginning.

The rate for the process (1) is given by

$$
\Gamma_{1}=\frac{G^{2}}{2 \pi^{5}}\left(q_{e} B_{m}\right)\left[\frac{2 q_{u} B_{m} q_{d} B_{m}}{5}\right]^{1 / 2} \cos ^{2} \theta_{c} T^{4} \frac{\mu_{u} \mu_{e}}{p_{F_{u}} p_{F_{e}}}
$$




$$
\int_{-\infty}^{+\infty} \int_{-\infty}^{+\infty}\left[x_{u}+x_{e}+\frac{\mu_{d}-\mu_{u}-\mu_{e}}{T}\right]^{2} f\left(x_{u}\right) f\left(x_{e}\right) d x_{u} d x_{e}
$$

where $q_{u}$ and $q_{d}$ are respectively the charge carried by $u$ and $d$-quarks.

Similarly the rate for the process (2) is given by

$$
\begin{aligned}
\Gamma_{2}= & \frac{G^{2}}{2 \pi^{5}}\left(q_{e} B_{m}\right)\left[\frac{2 q_{u} B_{m} q_{d} B_{m}}{5}\right]^{1 / 2} \cos ^{2} \theta_{c} T^{4} \\
& \int_{-\infty}^{+\infty} \int_{-\infty}^{+\infty}\left[x_{u}+x_{e}+\frac{\mu_{u}+\mu_{e}-\mu_{d}}{T}\right]^{2} f\left(x_{u}\right) f\left(x_{e}\right) d x_{u} d x_{e}
\end{aligned}
$$

In the present scenario, the rates for the processes (3) and (4) can not be obtained by simply replacing $d$-quark parameters with the corresponding $s$-quark values and $\cos ^{2} \theta_{c}$ by $\sin ^{2} \theta_{c}$ in eqns.(22) and (23) respectively. Since $s$-quarks are not affected by the magnetic field, we have to evaluate $\Gamma_{3}$ and $\Gamma_{4}$ with zero field plane wave solution for $s$-quark and modified Dirac spinors for all other charged species. Then we have the expressions for rates of the processes given by eqns.(3) and (4) for zero Landau quantum number

$$
\begin{aligned}
\Gamma_{3}= & \frac{5 G^{2}}{16 \pi^{7}}\left(q_{e} B_{m} q_{u} B_{m}\right) T^{4} \sin ^{2} \theta_{c} \int_{-\infty}^{+\infty} \int_{-\infty}^{+\infty} f\left(x_{u}\right) f\left(x_{e}\right) \\
& \left(x_{u}+x_{e}+\frac{\mu_{s}-\mu_{u}-\mu_{e}}{T}\right)^{2} d x_{u} d x_{e}
\end{aligned}
$$

and

$$
\begin{aligned}
\Gamma_{4}= & \frac{5 G^{2}}{16 \pi^{7}}\left(q_{e} B_{m} q_{u} B_{m}\right) T^{4} \sin ^{2} \theta_{c} \int_{-\infty}^{+\infty} \int_{-\infty}^{+\infty} f\left(x_{u}\right) f\left(x_{e}\right) \\
& \left(x_{u}+x_{e}-\frac{\mu_{s}-\mu_{u}-\mu_{e}}{T}\right)^{2} d x_{u} d x_{e}
\end{aligned}
$$

respectively.

The rate for the process (5) is given by

$$
\Gamma_{5}=\frac{9 G^{2}}{40 \pi^{6}}\left(q_{e} B_{m}\right)^{2} \cos ^{2} \theta_{c} \sin ^{2} \theta_{c} \sqrt{\frac{5}{6}} \frac{\mu_{u} \mu_{d}}{p_{F_{u}} p_{F_{d}}}
$$




$$
\begin{aligned}
& T\left(1-\frac{p_{F_{u}} p_{F_{d}}}{\mu_{u} \mu_{d}}\right) \int_{x_{u}=-\infty}^{+\infty} d x_{u} f\left(x_{u}\right) f\left(-x_{u}-x_{s}+\frac{\mu_{d}+\mu_{s}}{T}\right) \\
& \exp \left(-\frac{3}{5 q_{e} B_{m}} p_{s_{x}}^{2}\right) \exp \left(-\frac{3}{2 q_{e} B_{m}} p_{s_{y}}^{2}\right)\left[1-\frac{\left(p_{F_{u}}+p_{F_{d}}-p_{s_{z}}\right) p_{s_{z}}}{\mu_{u} E_{s}}\right] f\left(x_{s}\right) d^{3} p_{s}
\end{aligned}
$$

where $d^{3} p_{s}=p_{s}^{2} d p_{s} \sin \theta d \theta d \phi$. Since $E_{s}=\sqrt{p_{s}^{2}+m_{s}^{2}}$, we have

$$
\begin{gathered}
d^{3} p_{s}=p_{s} E_{s} d E_{s} \sin \theta d \theta d \phi \\
p_{s_{x}}=p_{s} \sin \theta \cos \phi \\
p_{s_{y}}=p_{s} \sin \theta \sin \phi
\end{gathered}
$$

and

$$
p_{s_{z}}=p_{s} \cos \theta
$$

To evaluate this integral we use $x_{s}=\left(\mu_{s}-E_{s}\right) / T$ as the new integration variable instead of $E_{3}$. Then we have

$$
\begin{aligned}
\Gamma_{5}= & \frac{9 G^{2}}{40 \pi^{6}}\left(q_{u} B_{m}\right)^{2} \cos ^{2} \theta_{c} \sin ^{2} \theta_{c} \sqrt{\frac{5}{6}} \frac{\mu_{u} \mu_{d}}{p_{F_{u}} p_{F_{d}}} \\
& T^{2}\left(1-\frac{p_{F_{u}} p_{F_{d}}}{\mu_{u} \mu_{d}}\right) \int_{-\infty}^{+\infty} \int_{-\infty}^{+\infty} d x_{u} d x_{s} \int_{0}^{\pi} \sin \theta d \theta \int_{0}^{2 \pi} d \phi \\
& f\left(x_{u}\right) f\left(x_{s}\right) f\left(-x_{u}-x_{s}+\frac{\mu_{d}+\mu_{s}}{T}\right)\left[1-\frac{\left(p_{F_{u}}+p_{F_{d}}-p_{s_{z}}\right) p_{s_{z}}}{\mu_{s} E_{s}}\right] \\
& \exp \left(-\frac{3}{5 q_{e} B_{m}} p_{s_{x}}^{2}\right) \exp \left(-\frac{3}{2 q_{e} B_{m}} p_{s_{y}}^{2}\right) p_{s} E_{s}
\end{aligned}
$$

The numerical evaluation of this multi-dimensional integral can be made little bit easier (less time consuming job in computer) by assuming $E_{3} \simeq \mu_{s}$ and $p_{s} \simeq p_{F_{s}}$ in those parts of this integral where $\theta, \phi$ and $E_{3}$ or $p_{3}$ are coupled together. The rate for reverse reaction is as usual given by eqn.(19). 
To study chemical evolution of the system, we have numerically solved the sets of kinetic equations given by eqns.(6) and (7). As has already been mentioned, we have considered three possible scenarios: $(i) B_{m}=0$, $(i i) B_{m}^{(c)(e)}<B_{m}<B_{m}^{(c)(u, d)}$ and $(i i i) B_{m}^{(c)(u, d)}<B_{m}<B_{m}^{(c)(s)}$. The initial conditions are $Y_{u}(t=0)=1.96$ and $Y_{d}(t=0)=1.04$ for the fractional abundances of $u$ and $d$-quarks. The rates of various processes (eqns.(1)-(5)) are also evaluated numerically and finally the charge neutrality and baryon number conservation conditions (eqns.(8) and (9)) are used to relate $Y_{s}(t)$ and $Y_{e}(t)$, the fractional abundances for $s$-quark and electron respectively with that of $u$ and $d$ quarks. In fig.(1) we have plotted time evolution of the fractional abundances $Y_{i}$ for $i=u, d, s$, and $e$ in the field free case. This figure shows that ultimately fractional abundances for $u, d, s$-quarks and electron saturate to their equilibrium values. Which is the chemical equilibrium condition among the participants. We have further noticed, that as the current mass $m_{s}$ for $s$-quark approaches the current mass of $u$ and $d$-quarks, the equilibrium values for fractional abundances of all the three types of quarks ultimately saturates to a constant value $\approx 1$ and the electron abundance $Y_{e} \approx 0$. We have also investigated the sensitivity of initial conditions. We have considered a wide variation of proton fraction- from almost pure neutron matter (proton fraction $x \approx 0.001$ ) to symmetric nuclear matter $(x=0.5)$. The qualitative nature of our results do not change with the change of initial condition. The change in initial condition only causes a change in saturation time scale. Now we would like to see whether such theoretical observation supports the speculation of Witten. We have verified whether the system in $\beta$-equilibrium is energetically stable or not. To do that, we have followed the following steps. (i) Using these equilibrium values for various components $(i=u, d, s$, and $e)$ we have 
obtained the equation of states for the quark matter system with $B_{m}=0$, (ii) using the equation of state we have solved TOV equation and obtained the mass of the quark core, which is $\sim 0.4 M_{\odot}$ for the central density $4 n_{0}$ and the corresponding radius is $R=3.8 \mathrm{Km}$. To obtain the mass of quark core in the field free case, we assume that the deconfinement transition is first order in nature. The corresponding critical density for a typical bag parameter value $B^{1 / 4}=160 \mathrm{MeV}$ is $n_{c} \approx 2.1 n_{0}$. It has been observed that the mass of the core increases in presence of a magnetic field where as the radius of the core decreases, i.e. the core becomes more compact in presence of strong magnetic fields [26]. To investigate the stability of such a bulk quark matter, we have calculated the energy per baryon using the relation

$$
\frac{\epsilon}{n_{B}}=\frac{1}{n_{B}}\left(\sum_{i=u, d, s, e} \epsilon_{i}+\epsilon_{\mathrm{mag} .}\right)
$$

where $\epsilon_{i}$ is the internal energy density of the species $i$ and $\epsilon_{\text {mag. }}=B_{m}^{2} /\left(8 \pi^{2}\right)$ is the magnetic energy density, which is zero in the field free case. Using the saturation values for abundances for various components, we have seen that $<\epsilon_{Q M} / n_{B}<$ $M_{n}<\epsilon_{N M} / n_{B}$. Which indicates that the chemical evolution of the system in field free case leads to a scenario which is energetically more stable than nuclear matter and the binding energy of the system is negative. Therefore, the observation is in agreement with the speculation of Witten. In fig.(2) we have plotted the variation of fractional abundances for various species with time for $B_{m}=10^{14} \mathrm{G}$, so that only electrons are affected. In the present scenario the deconfinement transition from hadronic matter to quark matter can not be first order. We assume the transition is metal-insulator type second order in nature. In this particular case, unlike a mixed phase of quark matter and hadronic matter in a first order quark hadron phase transition, we have a sharp interface between nuclear matter crust and quark matter 
core. At the sharp boundary all the thermodynamic variables change continuously, i.e. $P_{N M}=P_{Q M}, \epsilon_{N M}=\epsilon_{Q M}, \mu_{p}=2 \mu_{u}+\mu_{d}$ and $\mu_{n}=\mu_{u}+2 \mu_{d}$. From these relations, we obtain the variation of critical density with the strength of magnetic field. In fig.(3) we have shown this variation. The corresponding bag parameter also changes with the strength of magnetic field. The dashed curve in fig.(3) shows the variation of $B^{1 / 4}$ with the strength of magnetic field. In both the curves, up to $B_{m}=10^{3} B_{m}^{(c)(e)}$ only electrons are affected beyond which we have considered the quantum mechanical effect of strong magnetic field on all the charged species except $s$-quark. In the nuclear matter sector, we have considered a $\sigma-\omega$ exchange type of mean field model [24]. These curves show that both the quantities remain almost constant (equal to their values for field free case) up to $B_{m}=10^{17} \mathrm{G}$, beyond which they become extremely large. As it is obvious from fig.(2) that in this particular case, the net production of $s$-quark starts after $10^{-14} \mathrm{sec}$, which is much earlier than the field free case, but $Y_{s}$ goes to zero by $10^{-10}$ sec and then it remains zero for ever. It is also obvious from the figure that in this case $d$ quark abundance also goes to zero by the same time scale and in the chemical equilibrium condition it remains zero. The system in chemical equilibrium is therefore mainly consists of $u$-quarks and electrons. If we translate this scenario to the case of a neutron star, this is equivalent to a proton rich core. As a consequence direct URCA process will play the main role in the cooling process by neutrino emission from this region, provided it is energetically stable [27]. To study the stability of such abnormal system, we have calculated internal energy per unit volume. Near nuclear density it is $\sim 4.39 \times 10^{14} \mathrm{MeV}^{4}$, which corresponds to free energy per baryon $>>M_{n}$ and also $>>$ energy per baryon for hadronic matter. The magnetic energy density 
is negligibly small compared to internal energy density of the system unless the magnetic field is $>10^{19} \mathrm{G}$. In this particular case, the overall energy of the system is therefore equal to the kinetic energy of the system. This indicates that the system is therefore energetically unstable if the magnetic field affects electrons present in the system. In fig.(4) we have plotted the abundances for various species against time for $B_{m}=5 \times 10^{16} \mathrm{G}$. In this case all the charged particles except $s$-quarks are affected. We have seen that $s$-quark production is absolutely forbidden in this particular scenario. The system behaves like a neutron rich (rich in $d$-quark) core. In this case free energy per unit volume is $\approx 4.9 \times 10^{12} \mathrm{MeV}^{4}$ near normal nuclear density, which again corresponds to energy per baryon $>>M_{n}$ and also $>>$ energy per baryon for hadronic matter. Therefore, in this case also, the system is energetically unstable. We have also noticed that the presence of a small percentage of $\lambda$-hyperons in the core region before phase transition does not change the conclusions. In figs.(5) and (6) we have plotted the abundances for the species with time for the presence of $\approx 25 \% \lambda$-hyperon before phase transition to quark matter for $B_{m}=0$ and $B_{m}=$ $5 \times 10^{16} \mathrm{G}$ respectively. We have noticed that for the field free case, the time evolution leads to a stable configuration, where as for the presence of strong magnetic field the system becomes energetically unstable in the chemical equilibrium condition. Therefore the presence of $\lambda$-hyperons in hadronic matter (which is most likely if the density of hadronic matter exceeds a few times normal nuclear density) before deconfinement transition does not change our conclusion. Next we shall consider the effect of neutrino trapping on the stability of quark matter core in presence of strong magnetic fields in the next section. 


\section{BASIC FORMALISM: WITH DEGENERATE NEUTRINOS}

In this section we shall develop a formalism to investigate the chemical evolution of the system considering the effect of neutrino degeneracy on the weak reaction rates for the field free case and also in presence of a strong magnetic field. Assuming the formation of a neutron star immediately after supernova explosion, then at the beginning the matter within the remnant, particularly at the central region is extremely lepton rich (neutrino trapped). Such an object is called proto-neutron star. Normally it takes a few second to convert a proto-neutron star to a neutron star by the emission of trapped neutrinos. If the density of such matter exceeds $3-4 n_{0}$, a deconfinement transition to neutrino trapped quark matter can occur at the core region. To study the chemical evolution of this neutrino trapped quark matter, we have to evaluate the rates for the processes

$$
\begin{aligned}
& d+\nu_{e} \leftrightarrow u+e^{-} \\
& s+\nu_{e} \leftrightarrow u+e^{-}
\end{aligned}
$$

and the direct and reverse reactions given by

$$
u+d \leftrightarrow u+s
$$

In the case of trapped neutrinos in a degenerate quark matter we have to solve the differential equations

$$
\frac{d Y_{u}}{d t}=-\frac{1}{n_{B}}\left[\Gamma_{1}^{\prime}+\Gamma_{2}^{\prime}\right]
$$

and 


$$
\frac{d Y_{d}}{d t}=\frac{1}{n_{B}}\left[\Gamma_{1}^{\prime}-\Gamma_{3}^{\prime}\right]
$$

We also have to consider the total lepton number conservation, given by

$$
Y_{l}=Y_{e}+Y_{\nu} \simeq 0.4
$$

The magic number 0.4 is conventionally used in the numerical computation of supernova explosion. The baryon number conservation and charge neutrality conditions are also used to obtain fractional abundances of all the species present in the system. In eqns.(36) and (37), $\Gamma_{i}^{\prime}$ 's are the rates of the processes (33)-(35).

\section{Rate of Weak Processes: $B_{m}=0$}

In the case of degenerate neutrinos, the neutrino chemical potential $\mu_{\nu} \neq 0$ and has to be evaluated from eqn.(38). The rates for the processes given by eqns.(33) and (34) are given by

$$
\Gamma_{1}^{\prime}=\frac{G^{2} \cos ^{2} \theta_{c}}{4 \pi^{5}} T^{3} \xi_{d}^{\prime}\left(4 \pi^{2}+\xi_{d}^{\prime 2}\right) J\left(\mu_{u}, \mu_{e}, \mu_{d}, \mu_{\nu}\right)
$$

and

$$
\begin{aligned}
\Gamma_{2}^{\prime}= & \frac{3 G^{2}}{4 \pi^{5}} \sin ^{2} \theta_{c} T^{3}\left(1-\exp \left(-\xi_{s}^{\prime}\right)\right) \int_{-\infty}^{\left(\mu_{s}-m_{s}\right) / T} d x \\
& \frac{\left(x+\xi_{s}^{\prime}\right)^{2}+\pi^{2}}{\left(1+e^{x}\right)\left(1+e^{-}\left(x+\xi_{s}\right)\right)} J\left(\mu_{u}-T x, \mu_{e}, \mu_{s}, \mu_{\nu}\right)
\end{aligned}
$$

Finally the effective rate for the process (35) is given by

$$
\begin{aligned}
\Gamma_{3}^{\prime}= & \frac{9}{2 \pi^{5}} G^{2} \cos ^{2} \theta_{c} \sin ^{2} \theta_{c} T^{3}\left(1-\exp \left(-\xi_{s}^{\prime}\right)\right) \\
& \int_{-\infty}^{\left(\mu_{s}-m_{s}\right) / T} d x \frac{(x+\xi)^{2}+\pi^{2}}{\left(1+e^{x}\right)\left(1+e^{-(x+\xi)}\right)} J\left(\mu_{u}, \mu_{s}-T x, \mu_{u}, \mu_{d}\right)
\end{aligned}
$$




\section{Rate of Weak Processes: $B_{m} \neq 0$}

Now we shall consider the weak process in the degenerate quark matter in presence of a strong magnetic field and also consider the effect of neutrino neutrino trapping. As before, we first assume that $B_{m}^{(c)(e)}<B_{m}<B_{m}^{(c)(u, d)}$, so that only electrons are affected quantum mechanically by the magnetic field. In this case the effective rate for the process (33) is given by

$$
\begin{gathered}
\Gamma_{1}^{\prime}=\frac{G^{2} \cos ^{2} \theta_{c}}{\pi^{3}}\left(T q_{e} B_{m}\right) \sum_{\nu=0}^{\infty}\left(\frac{p_{F_{u}}}{p_{F_{e}}}\right) \mu_{u} \mu_{e} \int_{x_{u}=0}^{\infty} d x_{u} \int_{0}^{\infty} d E_{2} \frac{1}{\left[\exp \left(-E_{2}^{\prime} / T-x_{u}\right)+1\right]} \\
E_{2}^{\prime} f\left(x_{u}\right)\left[1+\exp \left(-\left(E_{2}-\mu_{\nu}\right) / T\right)\right]
\end{gathered}
$$

where $E_{2}^{\prime}=E_{2}-\left(\mu_{u}+\mu_{e}-\mu_{d}\right)$.

To obtain rate for the process (34), we have to replace all the parameters of $d$-quark by the corresponding $s$-quark parameters and $\cos ^{2} \theta_{c}$ has to be replaced by $\sin ^{2} \theta_{c}$. In this case $E_{2}^{\prime}=E_{2}-\left(\mu_{u}+\mu_{e}-\mu_{s}\right)$. The rate for the process (35) is given by eqn.(41), which is the expression for field free case.

When the magnetic field strength $B_{m}>B_{m}^{(c)(u, d)}$ but less than $B_{m}^{(c)(s)}$, all the charged species, except $s$-quarks are affected by the strong magnetic field. As has been mentioned before, compact analytical expressions for rates can only be obtained if we assume that only the zeroth Landau levels are populated for all the magnetically affected charged particle components. In this case the rate for the process (33) is given by

$$
\begin{aligned}
\Gamma_{1}^{\prime}= & \frac{3 G^{2}}{2 \pi^{4}} q_{e} B_{m}\left(\frac{q_{u} B_{m} q_{d} B_{m}}{17}\right) \cos ^{2} \theta_{c} \frac{\mu_{u} \mu_{e}}{p_{F_{u}} p_{F_{e}}} T \\
& \int_{x_{e}=-\infty}^{+\infty} \int_{E_{2}=0}^{\infty} \int_{\theta=0}^{\pi} \int_{\phi=0}^{2 \pi}\left(1-\frac{p_{F_{d}} p_{2_{z}}}{\mu_{d} E_{2}}\right) \exp \left(-p_{2_{x}}^{2} / 2 q_{e} B_{m}\right) \exp \left(-9 p_{2_{y}}^{2} / 17 q_{e} B_{m}\right) \\
& {\left[1+\exp \left(-\left(E_{2}-\mu_{\nu}\right) / T\right)\right] E_{2}^{2} \frac{1}{\left[1+\exp \left(-x_{e}-E_{2}^{\prime} / T\right)\right]} f\left(x_{e}\right) d x_{e} d E_{2} \sin \theta d \theta d \phi }
\end{aligned}
$$


As before the rate for the process (34) can not be obtained simply by replacing $d$-quark parameters by the corresponding $s$-quark values and $\cos ^{2} \theta_{c}$ by $\sin ^{2} \theta_{c}$. In this case the rate is given by

$$
\begin{gathered}
\Gamma_{2}^{\prime}=\frac{5 G^{2}}{16 \pi^{7}}\left(q_{e} B_{m} q_{u} B_{m}\right)\left(\frac{\mu_{u}}{p_{F_{u}}}\right)\left(\frac{\mu_{e}}{p_{F_{e}}}\right) T^{4} I \sin ^{2} \theta_{c} \\
I=\int_{-\infty}^{+\infty} \int_{-\infty}^{+\infty}\left(x_{u}+x_{e}-\frac{\mu_{s}-\mu_{u}-\mu_{e}}{T}\right)^{2} f\left(x_{u}\right) f\left(x_{e}\right) d x_{u} d x_{e}
\end{gathered}
$$

The rate for the process (35) is given by the combination of eqns.(31) and (19).

In the degenerate neutrino case, we have plotted the time evolution of fractional abundances for various species in the filed free case in fig.(7). In this case the system is marginally stable, internal energy density is $\approx 6.25 \times 10^{9} \mathrm{MeV}^{4}$. Since it takes a few second to convert such a system to conventional system of SQM with non-degenerate neutrinos by neutrino emission, we may conclude that it is possible to have an energetically stable quark matter core from the deconfinement transition at the central region of a compact proto-neutron star. In the case when $B_{m}^{(c)(e)}<B_{m}<B_{m}^{(c)(u, d)}$, i.e., only electrons are affected, we have seen that almost all the neutrinos are absorbed in the system momentarily and to keep lepton number constant, electron density increases by several orders of magnitude. As a consequence of charge neutrality condition, the $u$-quark density also increases by the same orders of magnitude. But the conservation of baryon number of the system causes decrease in $d$-quark density almost by the same orders of magnitude. The situation is happened to be the most unphysical one. In this case the binding energy calculation shows that the system is extremely unstable. In this case internal energy density is $\approx 10^{18} \mathrm{MeV}^{4}$ near normal nuclear density. In the case of strong magnetic field $\left(B_{m}^{(c)(u, d)}<B_{m}<B_{m}^{(c)(s)}\right)$, when all the charged components except $s$-quark are 
affected, we have shown the time dependence of fractional abundances in fig.(8). In this case also $s$-quarks are never produced and the conclusion does not change if there are a few $\lambda$-hyperons at the central region of proto-neutron star before phase transition. We have also seen that electron density goes up by several orders of magnitude, as a consequence $u$-quark density also increases and the system behaves like a neutrino trapped proton core (if we translate it to proto-neutron star without quark core). The scenario is also unphysical in this case. The internal energy of the system is $\approx 4.376 \times 10^{17} \mathrm{MeV}^{4}$. Therefore, we can conclude that in presence of a strong magnetic field, with trapped neutrinos, the formation of quark matter core by deconfinement transition at the central region of a compact neutron star is also energetically forbidden.

\section{CONCLUSIONS AND DISCUSSIONS}

We have studied the chemical evolution of quark matter core in presence of a strong magnetic field and compared our results with that of field free case. We have noticed that in presence of a magnetic field of strength at least greater than $B_{m}^{(c)(e)}$, the critical field strength to populate Landau levels for electrons, quark matter core in compact neutron star or proto-neutron star is not possible. The quark matter core is energetically unstable with respect to normal hadronic matter in presence of such strong magnetic field. On the other hand if a charge neutral SQM in beta equilibrium is placed in an external strong magnetic field the system becomes energetically more stable compared to field free case. We have also seen that the presence of $\lambda$-hyperons at the core of a neutron star before phase transition to deconfined quark matter does not alter the characteristic of newborn quark matter core in presence of strong 
magnetic field. We have also noticed that our conclusion is insensitive with the numerical value of proton fraction. Therefore, the final conclusion is that the quark matter core is not at all possible in a compact neutron star or proto-neutron star if the magnetic field at the central region exceeds at least the critical value for electron to populate Landau levels. In an earlier work we showed that a first order QCD phase transition from nuclear matter to quark matter is not possible in presence of a strong magnetic field [4,5, 16]. Even if a metal-insulator type second order phase transition is possible, the stability criteria does not allow the formation of quark matter core in a compact neutron star or in a proto-neutron star. However, such a phase transition is possible in the case of a very old neutron star by matter accretion, during slowing down of a pulser which may cause further contraction of neutron star, nucleation of SQM by some external sources or by neutrino sparking. Instead of a phase transition to quark matter it is possible to have kaon condensed hadronic matter at the core region. It would be interesting to see what is the effect of strong magnetic field on such condensed state. It can also be a di-quark matter. It would also be interesting to study the effect of strong magnetic field on such matter. 


\section{REFERENCES}

[1] E. Witten, Phys. Rev. D30, 272 (1984).

[2] C. Alcock, E. Farhi and A.V. Olinto, Astrophys. J. 310, 261 (1986).

[3] P. Haensel, J.D. Zdunik and R. Schaeffer, Astron. Astrophys. 160, 12 (1986).

[4] S. Chakrabarty, Phys. Rev. D43, 627 (1991) and references their in.

[5] S. Chakrabarty, Phys. Rev. D51, 4591 (1995).

[6] J. Madsen, Phys. Rev. D47, 325 (1993).

[7] H. Heiselberg, J. Madsen and K. Riisager, Physica Scr. 34, 556 (1986).

[8] N.K. Glendenning, astro-ph/9803067, astro-ph/9803070, astro-ph/9803074-and references therein.

[9] H. Heiselberg and M. Hjorth-Jensen, astro-ph/9801187.

[10] J.E. Horvath and H. Vucetich, astro-ph/9809385.

[11] G. Chanmugam, Ann. Rev. Astron. Astrophys. 30, 143 (1992).

[12] M.G. Baring and A.K. Harding, astro-ph/9809115.

[13] C. Kouveliotou et al, astro-ph/9809140.

[14] D. Lai and S.L. Shapiro, Astrophys. J. 383, 745 (1991).

[15] S. Chandrasekhar and E. Fermi, Astrophys. J. 118, 116 (1953).

[16] S. Chakrabarty, Phys. Rev. D54, 1306 (1996).

[17] T. Ghosh and S. Chakrabarty, Phys. Rev. D (communicated). 
[18] S. Chakrabarty, Astron. Space Sci 213, 121 (1994); S. Chakrabarty and A.K. Goyal, Mod. Phys. Lett. A9, 3611 (1994).

[19] J.S. Heyl and L. Hernquist, hep-th/9607124.

[20] A. Vilenkin, Astrophys. J. 451, 700 (1995).

[21] C.J. Horwitz and G.Li, Indiana Univ. preprint, 1997.

[22] D. Lai and Yong-Zhong Qian, astro-ph/9712043.

[23] G. Raffelt, astro-ph/9808099.

[24] S. Chakrabarty, D. Bandopadhyay and S. Pal, Phys. Rev. Lett. 78, 2898 (1997).

[25] S. Chakrabarty, D. Bandopadhyay and S. Pal, Int. J. Mod. Phys. A13, 295 (1998).

[26] S. Chakrabarty and P.K. Sahu, Phys. Rev. D53, 4687 (1996).

[27] D. Bandopadhyaya, S. Chakrabarty, P. De and S. Pal, Phys. Rev. D, rapid communication 1998 (in press). 
This figure "fig1-1.png" is available in "png" format from: http://arxiv.org/ps/astro-ph/9811243v1 
This figure "fig1-2.png" is available in "png" format from: http://arxiv.org/ps/astro-ph/9811243v1 
This figure "fig1-3.png" is available in "png" format from: http://arxiv.org/ps/astro-ph/9811243v1 
This figure "fig1-4.png" is available in "png" format from: http://arxiv.org/ps/astro-ph/9811243v1 
This figure "fig1-5.png" is available in "png" format from: http://arxiv.org/ps/astro-ph/9811243v1 
This figure "fig1-6.png" is available in "png" format from: http://arxiv.org/ps/astro-ph/9811243v1 
This figure "fig1-7.png" is available in "png" format from: http://arxiv.org/ps/astro-ph/9811243v1 
This figure "fig1-8.png" is available in "png" format from: http://arxiv.org/ps/astro-ph/9811243v1 